\title{
Requirements for CEC POP Machine Protection System
}

\author{
I. Pinayev
}

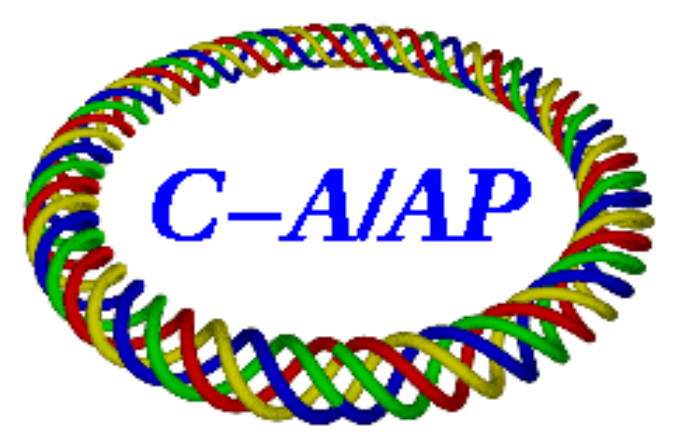

\section{Collider-Accelerator Department Brookhaven National Laboratory Upton, NY 11973}

\section{U.S. Department of Energy Office of Science, Office of Nuclear Physics}

Notice: This document has been authorized by employees of Brookhaven Science Associates, LLC under Contract No. DE-SC0012704 with the U.S. Department of Energy. The United States Government retains a nonexclusive, paid-up, irrevocable, world-wide license to publish or reproduce the published form of this document, or allow others to do so, for United States Government purposes. 


\section{REQUIREMENTS FOR CEC POP MACHINE PROTECTION SYSTEM}

The machine protection system should prevent damage of a vacuum chamber with missteered electron beam. For the evaluation purposes we will utilise the following beam electron parameters:

$\begin{array}{ll}\text { Energy } & -22 \mathrm{MeV} \\ \text { Maximal bunch charge } & -5 \mathrm{nC} \\ \text { Maximal repetition rate } & -78 \mathrm{kHz} \\ \text { Normalized emittance } & -5 \mathrm{~mm} \text { mrad } \\ \text { Minimal } \beta \text {-function } & -1 \mathrm{~m}\end{array}$

Vacuum system is build of steel, for which total stopping power of the $22 \mathrm{MeV}$ electron beam is about $2.8 \mathrm{MeV} \mathrm{g} / \mathrm{cm}^{2}$ [1]. For the steel density of $7.8 \mathrm{~g} / \mathrm{cm}^{3}$ specific losses (per unit of length) will be $21.8 \mathrm{MeV} / \mathrm{cm}$. For $2 \mathrm{MeV}$ electron beam stopping power is 1.4 $\mathrm{MeV} \mathrm{g} / \mathrm{cm}^{2}$ [1] and losses will be $10.9 \mathrm{MeV} / \mathrm{cm}$.

Maximal density of the electron current is defined by maximal current of Imax $=390 \mu \mathrm{A}$ and minimal beam size, which is assumed equal in both planes. At $\gamma=43$ the r.m.s. emittance will be $120 \mathrm{~nm}$ and r.m.s. beam size in each plane will be $\sigma=340 \mu$. The peak current density for Gaussian beam is equal to $I_{\max } / 2 \pi \sigma^{2}=0.054 \mathrm{~A} / \mathrm{cm}^{2}$, and volumetric power dissipation will be $1.17 \mathrm{MW} / \mathrm{cm}^{3}$. The volumetric heat capacity of iron is 3.5 $\mathrm{J} / \mathrm{cm}^{3} / \mathrm{K}$. Allowing temperature excursions up to $\Delta \mathrm{T}=200^{\circ} \mathrm{K}$ we can find the maximal reaction time of the machine protection system

$$
\mathrm{t}_{\max }=3.5 \times 200 / 1.17 \times 10^{6}=600 \mu \mathrm{s}
$$

It should be noted that the obtained result does not depend either beam has normal or shallow incidence on the surface.

Now we will estimate how much current loss we can withstand in long run. Essentially is the same limit below which we can perform any beam manipulations without destroying the vacuum chamber or diagnostics equipment. Because the transverse size of the electron beam is much smaller than the loss length $(1 \mathrm{~cm}$ for $22 \mathrm{MeV}$ beam, and $2 \mathrm{~mm}$ for $2 \mathrm{MeV}$ beam) we can consider the cylindrical geometry. Let consider the electron beam with radius $r$, and uniform current density $j=I / \pi r^{2}$. The volumetric heat dissipation will be $H=j L$, where $L$ is specific losses in the wall. Inside the cylinder of radius $x<r$ and length $l$ total generated heat will be $P=j L \pi x^{2} l$, and these power will be dissipated through conductance $P=-2 \pi x l k d T / d x$, where $\mathrm{k}$ is thermal conductivity (80 W/K/m for iron). Or $d T / d x=-j L x / 2 k$ and

$$
T(x)=T_{\max }-j L r^{2} / 4 k(x / r)^{2}=T_{\max }-I L / 4 \pi k(x / r)^{2}
$$

for $\mathrm{x} \leq \mathrm{r}$. For $\mathrm{x}>\mathrm{r}$ the amount of heat generated is fixed $P=I L l$ and temperature gradient is $d T / d x=-I L / 2 \pi k x$. Temperature dependence on distance is logarithmic, therefore we will assume that at some distance $R$ temperature is fixed $\mathrm{T}=0$. Then dependence will be $T(x)=I L \ln (R / x) / 2 \pi k$, and temperature at beam boundary will be $T(r)=I L \ln (R / r) / 2 \pi k$. Now we can find $T_{\max }$ :

$$
T_{\max }=I L / 4 \pi k+I L \ln (R / r) / 2 \pi k=I L / 4 \pi k(1+1 / 2 \ln (R / r))
$$


For $R$ we can choose triple loss length and for $r$ the smallest beam size. For this case

$$
T_{\text {max }} \approx I L / 4 k
$$

and assuming maximal temperature rise of $35^{\circ} \mathrm{C}$ the maximal allowable loss current is

$$
I_{\text {loss } \max }=4 \mathrm{k} T_{\max } / L=4 \times 0.8 \times 35 / 21.8 \times 10^{6}=5.1 \mu \mathrm{A}
$$

\section{REFERENCE}

1. http://physics.nist.gov/PhysRefData/Star/Text/ESTAR.html 